\title{
ANTARES search for neutrino flares from the direction of radio-bright blazars
}

Giulia Illuminati ${ }^{a, b, *}$ and Alexander Plavin ${ }^{c, d}$ on behalf of the ANTARES Collaboration
(a complete list of authors can be found at the end of the proceedings)

${ }^{a}$ INFN - Sezione di Bologna, Viale Berti-Pichat 6/2, 40127 Bologna, Italy

${ }^{b}$ Dipartimento di Fisica e Astronomia dell'Università, Viale Berti Pichat 6/2, 40127 Bologna, Italy

${ }^{c}$ Lebedev Physical Institute, Leninsky prospekt 53, 119991 Moscow, Russia

${ }^{d}$ Moscow Institute of Physics and Technology, Institutsky per. 9, Dolgoprudny, 141700, Russia

E-mail: giulia.illuminati3@unibo.it, alexander@plav.in

In 2017, a high-energy muon neutrino detected by IceCube was found positionally coincident with the direction of a known blazar, TXS 0506+056, in a state of enhanced $\gamma$-ray emission. Soon after, IceCube reported a compelling evidence for an earlier neutrino flare from the same direction found in the archival data, this time not accompanied by any observed electromagnetic activity. The IceCube findings suggest searching for flaring neutrino emissions from astrophysical sources, not necessarily accompanied by flares detected in $\gamma$-rays. The analysis presented in this contribution scans the events collected by the ANTARES neutrino telescope in 13 years of data taking in a search for clustering in space and time. The analysis method is based on an unbinned maximum likelihood approach. Generic Gaussian and Box profiles are assumed for the signal time emission, with both the central time and duration of the flare being free parameters in the likelihood maximization. The time-dependent approach is applied to the catalog of radio-bright blazars for which a promising directional correlation with IceCube muon tracks was recently reported [ApJ 894 (2020) 101, ApJ 908 (2021) 157].

$37^{\text {th }}$ International Cosmic Ray Conference (ICRC 2021)

July 12 th - 23rd, 2021

Online - Berlin, Germany

\footnotetext{
*Presenter
} 


\section{Introduction}

On 22 September 2017, the IceCube Collaboration detected a $300 \mathrm{TeV}$ muon neutrino, with arrival direction compatible with the position of a known blazar, TXS 0506+056, found in a flaring state at the time of the neutrino detection $[1,2]$. Triggered by this association, the IceCube Collaboration performed a search for clustering in time at the position of TXS $0506+056$ assuming two different generic profile shapes: a Gaussian-shaped time window and a Box-shaped time window [3]. The analysis yielded a $3.5 \sigma$ evidence for an excess of high-energy neutrino events, with respect to atmospheric backgrounds, at the position of the blazar between September 2014 and March 2015. No electromagnetic activity was observed during the neutrino flare.

Additional hints that blazars are neutrino source candidates were reported more recently, when a search for correlation between radio-selected blazars and very-high-energy track-like IceCube events resulted in a promising evidence of association [4], later confirmed in a second analysis that made use of lower-energy IceCube neutrinos [5]. A similar search for spatial correlation between the same radio-selected blazars and neutrinos detected by the ANTARES telescope has been performed and is presented in these proceedings [6].

The list of radio-bright blazars used in [4-6] corresponds to an all-sky sample of 3411 blazars, selected on the basis of their very-long-baseline interferometry (VLBI) radio flux, as those sources with a flux density integrated over VLBI images at $8 \mathrm{GHz}$ of at least $150 \mathrm{mJy}$. The blazar MG3 J225517+2409 (J2255+2410) - one of the most significant sources found in the ANTARES point-source stacking analysis [7] - is not included in the catalog due to the low measured flux. Here, the most up-to-date version of this catalog (version 2021b, available at the following link http: //astrogeo.org/ $\mathrm{rfc} /$ ) is employed in a search for time and space clustering of ANTARES events from the direction of the blazars. Only sources with a declination $\delta<40^{\circ}$ are employed in the analysis, for a total of 2774 investigated blazars. The search makes use of the ANTARES events detected between January 29, 2007 and February 29, 2020 (3845 day livetime) and selected for the 13-year ANTARES point-like source analysis [8], corresponding to a total of 10162 track-like and 225 shower-like events.

\section{Search Method and Expected Performance}

The search for neutrino flares relies on an unbinned time-dependent maximum likelihood method. The likelihood, that describes the ANTARES data in terms of signal and background probability density functions (PDFs), is defined as:

$$
\log \mathcal{L}_{\mathrm{s}+\mathrm{b}}=\sum_{\mathcal{J} \in\{t r, s h\}} \sum_{i \in \mathcal{J}} \log \left[\frac{\mu_{\text {sig }}^{\mathcal{J}}}{\mathcal{N}^{\mathcal{J}}} \mathcal{S}_{i}^{\mathcal{J}}+\frac{\mathcal{N}^{\mathcal{J}}-\mu_{\text {sig }}^{\mathcal{J}}}{\mathcal{N}^{\mathcal{J}}} \mathcal{B}_{i}^{\mathcal{J}}\right],
$$

where $\mathcal{S}_{i}^{\mathcal{J}}$ and $\mathcal{B}_{i}^{\mathcal{J}}$ are the values of the signal and background PDFs for the event $i$ in the sample $\mathcal{J}$ (tr for tracks, $s h$ for showers), while $\mu_{\text {sig }}^{\mathcal{J}}$ and $\mathcal{N}^{\mathcal{J}}$ are respectively the number of unknown signal events and the total number of data events in the $\mathcal{J}$ sample. The combined information of three parameters - direction, energy and detection time - is included in the definition of the PDFs in order to enhance the signal-to-background discrimination. The same definition of the direction and 
energy PDFs used in the 9-year ANTARES point-like source search [9] is employed. Regarding the time PDFs, two time profiles are tested for the signal emission, characterized by a Gaussian shape and a Box shape, and defined as:

$$
\mathcal{S}_{\text {Gaussian }}^{\text {time }}\left(t_{i}\right)=\frac{1}{\sqrt{2 \pi} \sigma_{t}} e^{\left(-\frac{\left(t_{i}-T_{0}\right)^{2}}{2 \sigma_{t}^{2}}\right)} \quad \mathcal{S}_{\mathrm{Box}}^{\text {time }}\left(t_{i}\right)=\left\{\begin{array}{ll}
\frac{1}{2 \sigma_{t}}, & \text { if }\left[T_{0}-\sigma_{t}\right] \leq t_{i} \leq\left[T_{0}+\sigma_{t}\right] ; \\
0, & \text { otherwise } ;
\end{array},\right.
$$

with $t_{i}$ being the detection time of the ANTARES event $i$, while $T_{0}$ and $\sigma_{t}$ being the unknown central time and duration of the flaring emission, respectively, both fitted in the likelihood maximisation. Concerning the background time profile, given the small expected contribution of a cosmic signal in the overall data set, this PDF is built using the time distribution of data events, ensuring a time profile proportional to the measured data. To avoid statistical fluctuations, this PDF is computed applying less stringent selection criteria than those of the final sample, using the same approach as in [10].

The likelihood of Equation 1 is maximised independently at the position of each investigated source leaving as free parameters the number of signal events $\mu_{\mathrm{sig}}=\mu_{\mathrm{sig}}^{t r}+\mu_{\mathrm{sig}}^{s h}$, the signal spectral index $\gamma$, the central time of the flare $T_{0}$, and the flare duration $\sigma_{t}$, providing the best-fit values $\hat{\mu}_{\text {sig }}$, $\hat{\gamma}, \hat{T}_{0}, \hat{\sigma}_{t}$ for each source candidate. In the maximisation, the spectral index can take values between 1.0 and 3.5, so to include the value predicted by the Fermi acceleration mechanism $(\gamma=2.0)$ and the softer best-fit spectral indices of the isotropic flux of high-energy cosmic neutrinos measured by the IceCube Collaboration (between $\gamma=2.92$ reported in [11] and $\gamma=2.28$ reported in [12]). As for the time-dependent parameters, $T_{0}$ can vary over the time range of the investigated ANTARES data (from 1st January, 2007 until 28th February, 2020), while $\sigma_{t}$ can take values between 1 day and 2000 days.

The test statistic of the analysis, $Q$, is derived from the likelihood as

$$
Q=2 \log \left[\frac{\mathcal{L}_{\mathrm{s}+\mathrm{b}}}{\mathcal{L}_{\mathrm{b}}} \times \frac{\hat{\sigma}_{t}}{\Delta T}\right],
$$

where $\mathcal{L}_{\mathrm{s}+\mathrm{b}}$ is the likelihood defined in Equation 1 evaluated with the best-fit values of the free parameters $\left(\mu_{\text {sig }}=\hat{\mu}_{\text {sig }}, \gamma=\hat{\gamma}, T_{0}=\hat{T}_{0}, \sigma_{t}=\hat{\sigma}_{t}\right), \mathcal{L}_{\mathrm{b}}$ is the likelihood evaluated in the backgroundonly case $\left(\mu_{\text {sig }}=0\right)$, while $\frac{\hat{\sigma}_{t}}{\Delta T}$ is a penalization term for short flares, with $\Delta T$ being the allowed time range for $T_{0}$. The penalization term accounts for the larger trial factor that should be associated to short flares since a larger number of short flares than of long ones can be accommodated in a given time range, as described in [13].

In order to estimate the significance of the best flare (with highest $Q$ ) of each investigated source, the $Q$-value obtained for the given flare is compared to the test statistic distribution obtained with background-only pseudo-experiments (PEs) at the corresponding source declination. The fraction of background-like PEs with a value of the test statistic larger than the observed $Q$ gives the significance ( $\mathrm{p}$-value) of the flare. The lowest obtained $\mathrm{p}$-value identifies the most significant flare of the search. Finally, a trial correction that accounts for the fact that many candidates have been investigated is applied. In particular, the lowest obtained p-value is compared to the distribution of the smallest p-values found when performing the same analysis on many background-only PEs. 
The expected performance of this approach in terms of discovery potential and sensitivity as a function of the duration of the flare is shown in Figure 1. The performance of the timedependent approach is compared to the one of the the time-integrated analysis, i.e. when the time information of the events is not considered, obtained using the same livetime [8]. While similar levels of sensitivities are obtained in the investigated range of flare durations, the time-dependent search performs better for flares shorter than $\sim 1000$ days in terms of discovery potential, with an improvement of a factor $\sim 2$ achieved for flares as short as 1 day.

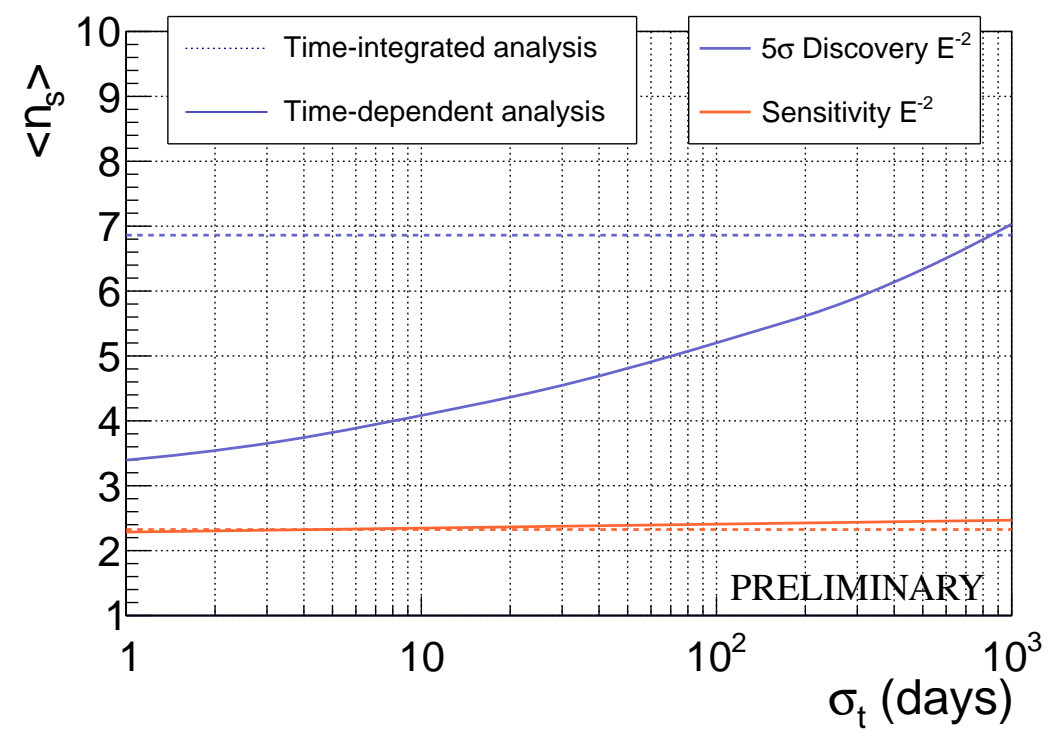

Figure 1: $5 \sigma$ discovery potential (blue) and 90\% C.L. sensitivity (orange) in terms of mean number of signal events as a function of the simulated flare duration for the time-integrated analysis (dotted) and for the time-dependent analysis (solid). The simulated source is at a declination of $\delta=-40^{\circ}$ and the flare is centered at $T_{0}[\mathrm{MJD}]=57000$. Similar results are obtained for different source declinations and central times.

\section{Results}

The search applied to the 2774 radio-bright blazars results in the p-value distributions shown in Figure 2. A pre-trial significance of over $3 \sigma$ for at least one of the tested time profile has been obtained for seven sources, indicated in Figure 2 and listed in Table 1, together with the corresponding best-fit values of the free parameters. The same source, J1500-2358, shows the lowest pre-trial p-value using both time profiles. The pre-trial significance of $3.3 \sigma(3.4 \sigma)$ obtained for the Gaussian-shape (Box-shape) assumption for J1500-2358 corresponds to a post-trial p-value of $56 \%(40 \%)$. The weighted time distribution of the ANTARES events close to J1500-2358 is shown in Figure 3. Only tracks (showers) within a distance of $5^{\circ}\left(10^{\circ}\right)$ from J1500-2358 are included in the plot. A higher weight is associated to events with smaller distance to the source and larger value of the energy estimator. 

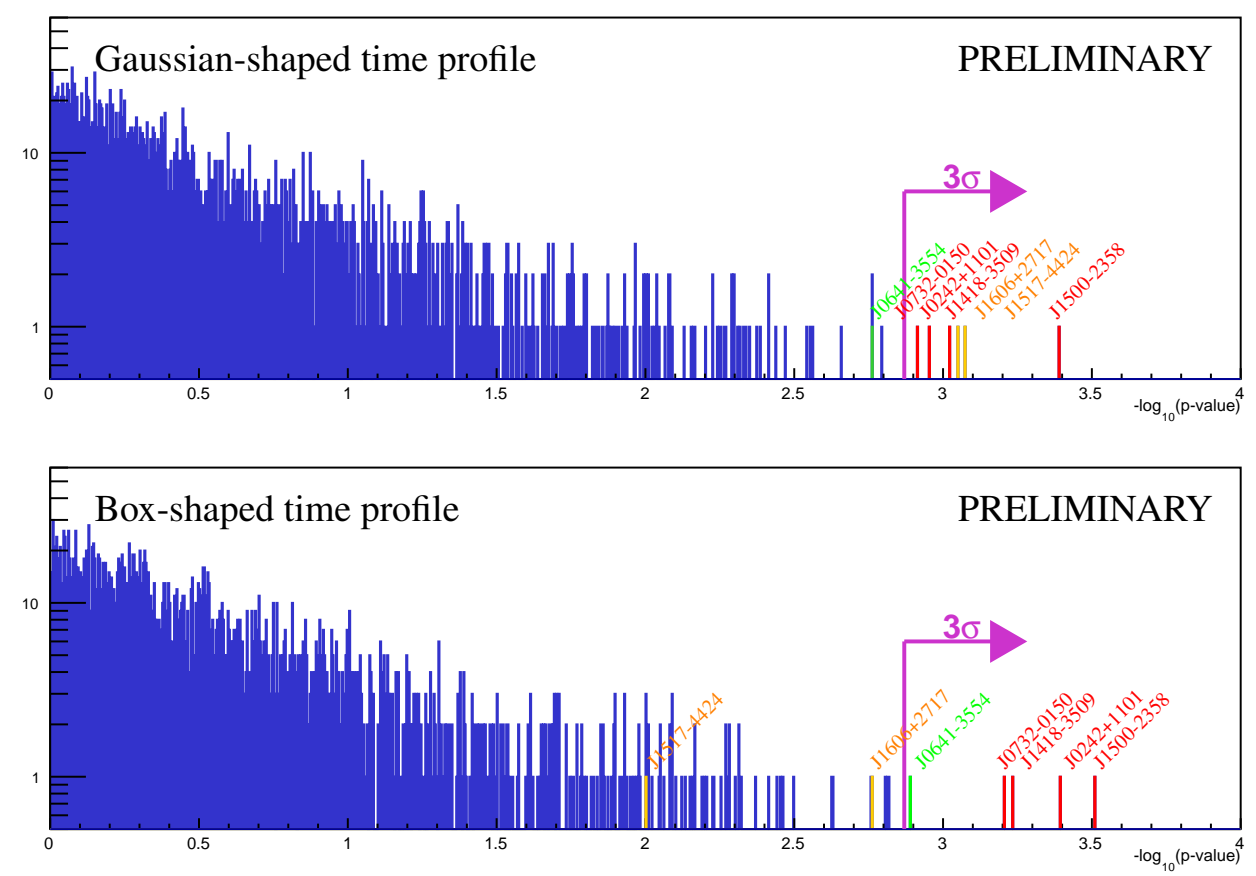

Figure 2: Distribution of the 2774 pre-trial p-values found at the investigated locations for the Gaussianshaped (top) and Box-shaped (bottom) signal time profile. The magenta arrow indicates the p-value corresponding to a pre-trial significance of at least $3 \sigma$. The name of the sources for which a pre-trial significance of over $3 \sigma$ has been obtained for both profile assumptions (red), or at least for one time profile (orange for Gaussian shape and green for Box shape) is reported above the corresponding p-value.

Table 1: List of radio-bright blazars for which a pre-trial significance of over $3 \sigma$ for at least one of the tested time profile (Gaussian-shaped and Box-shaped) has been obtained. The first three columns report the name and equatorial coordinates of the sources. The remaining columns summarise the results of the search in terms of best-fit central time of the flare $\hat{T}_{0}$, flare duration $\hat{\sigma}_{t}$, number of signal events $\hat{\mu}_{\text {sig }}$, spectral index $\hat{\gamma}$ and pre-trial p-value, for the Gaussian-shaped and Box-shaped signal time profile. The sources with over $3 \sigma$ pre-trial significance with both time profiles are highlighted in bold.

\begin{tabular}{|c|c|c|c|c|c|c|c|c|c|c|c|c|}
\hline \multicolumn{3}{|c|}{ Source } & \multicolumn{10}{|c|}{ Results } \\
\hline \multirow[t]{2}{*}{ Name } & \multirow{2}{*}{$\begin{array}{c}\delta \\
{[\mathrm{deg}]}\end{array}$} & \multirow{2}{*}{$\begin{array}{c}\alpha \\
{[\operatorname{deg}]}\end{array}$} & \multicolumn{5}{|c|}{ Gaussian-shaped time profile } & \multicolumn{5}{|c|}{ Box-shaped time profile } \\
\hline & & & $\begin{array}{c}\hat{T}_{0} \\
{[\mathrm{MJD}]}\end{array}$ & $\begin{array}{c}\hat{\sigma}_{t} \\
\text { [days] }\end{array}$ & $\hat{\mu}_{\text {sig }}$ & $\hat{\gamma}$ & p-value & $\begin{array}{c}\hat{T}_{0} \\
{[\mathrm{MJD}]}\end{array}$ & $\begin{array}{c}\hat{\sigma}_{t} \\
\text { [days] }\end{array}$ & $\hat{\mu}_{\text {sig }}$ & $\hat{\gamma}$ & p-value \\
\hline J1500-2358 & -24.0 & 225.2 & 55846 & 4 & 3.7 & 2.2 & 0.00041 & 55846 & 6 & 3.7 & 2.2 & 0.00031 \\
\hline $\mathrm{J} 1517-4424$ & -44.4 & 229.4 & 57761 & 361 & 7.2 & 3.5 & 0.00084 & 57366 & 529 & 5.3 & 3.5 & 0.0099 \\
\hline $\mathrm{J} 1606+2717$ & 27.3 & 241.7 & 58793 & 1 & 1.0 & 1.1 & 0.00089 & 58267 & 538 & 1.2 & 1.3 & 0.0017 \\
\hline J1418-3509 & -35.2 & 214.7 & 58119 & 12 & 3.6 & 3.3 & 0.00095 & 58119 & 14 & 3.8 & 3.3 & 0.00058 \\
\hline J0242+1101 & 11.0 & 40.6 & 56634 & 318 & 5.3 & 2.0 & 0.0011 & 56635 & 413 & 5.6 & 2.1 & 0.00040 \\
\hline J0732-0150 & 1.8 & 113.1 & 55794 & 82 & 4.9 & 3.5 & 0.0012 & 55813 & 117 & 5.2 & 3.5 & 0.00062 \\
\hline J0641-3554 & -35.9 & 100.3 & 58084 & 16 & 3.0 & 3.2 & 0.0017 & 58080 & 18 & 3.0 & 3.2 & 0.0013 \\
\hline
\end{tabular}



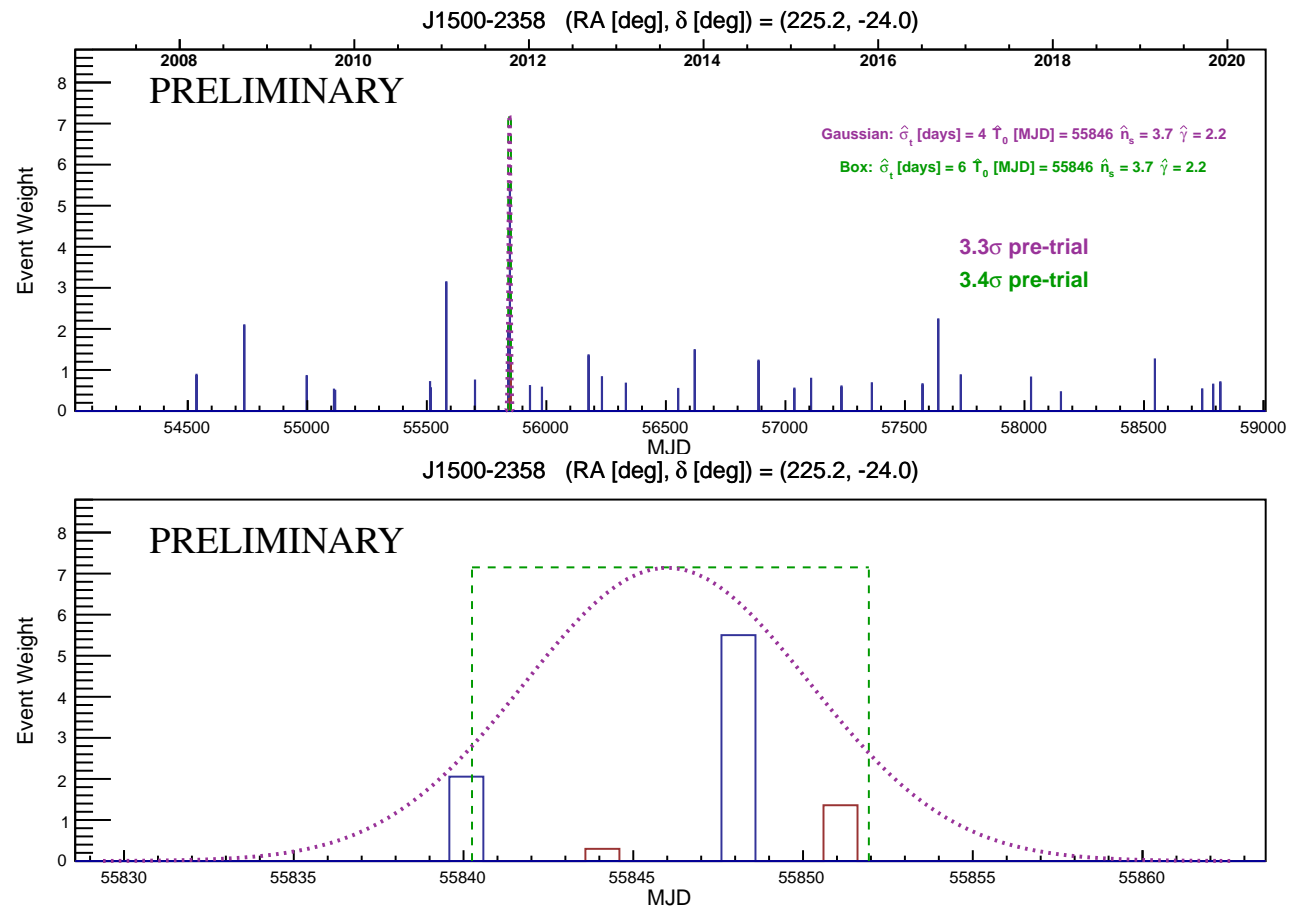

Figure 3: Weighted time distribution of the ANTARES events close to the location of J1500-2358. The top plot spans over the whole analysed time range, while the bottom plot shows the events within $3 \hat{\sigma}_{t}$ from $\hat{T}_{0}$ fitted with the Box-shaped profile. The green Box profile and the magenta Gaussian profile have been drawn using the best-fit values of $\hat{\sigma}_{t}$ and $\hat{T}_{0}$ found in each case. Tracks (showers) are shown in blue (red).

\subsection{The notable case of J0242+1101 (PKS 0239+108)}

As a follow-up study of the findings of this analysis, the obtained best-fit neutrino flares have been compared to the radio light-curves produced by the Owens Valley Radio Observatory [OVRO 14] for those sources of Table 1 for which radio data are available. The most interesting case is the one of the blazar J0242+1101 with a typical parsec-scale core-jet structure. Its largest flare observed in radio shows a notable overlap in time with the best-fit neutrino flare found in this analysis for the same source, as shown in Figure 4. In view of this intriguing observation, the time distribution of the public data of the Fermi $\gamma$-ray telescope and of the IceCube neutrino telescope compatible with the source direction have also been studied. The adaptive binned $\gamma$-ray light-curve, obtained from Fermi data using the method described in [15], for J0242+1101, is shown in Figure 4. Remarkably, the most significant Fermi $\gamma$-ray flare for this source happened during the flaring emission observed in radio and the period highlighted by the present analysis of ANTARES neutrinos. Finally, the time distribution of the IceCube tracks of the 10-year point-source sample [16] with direction compatible with the blazar position within the 50\% angular error reported by the IceCube Collaboration, is also shown. Only events with an angular uncertainty smaller than $10 \mathrm{deg}^{2}$ are depicted. While there is not evidence of time clustering of the IceCube events, a muon-neutrino-induced track with the notable high energy of $50 \mathrm{TeV}$ was detected during the flare. A dedicated analysis will be soon performed to estimate the chance probability of the association between radio, $\gamma$-ray and neutrino observations. It is also worth mentioning that $\mathrm{J} 0242+1101$ has been analysed with a 
time-independent approach in the 13-year ANTARES point-like source analysis [8], and was found to be the most significant of the 121 investigated sources.

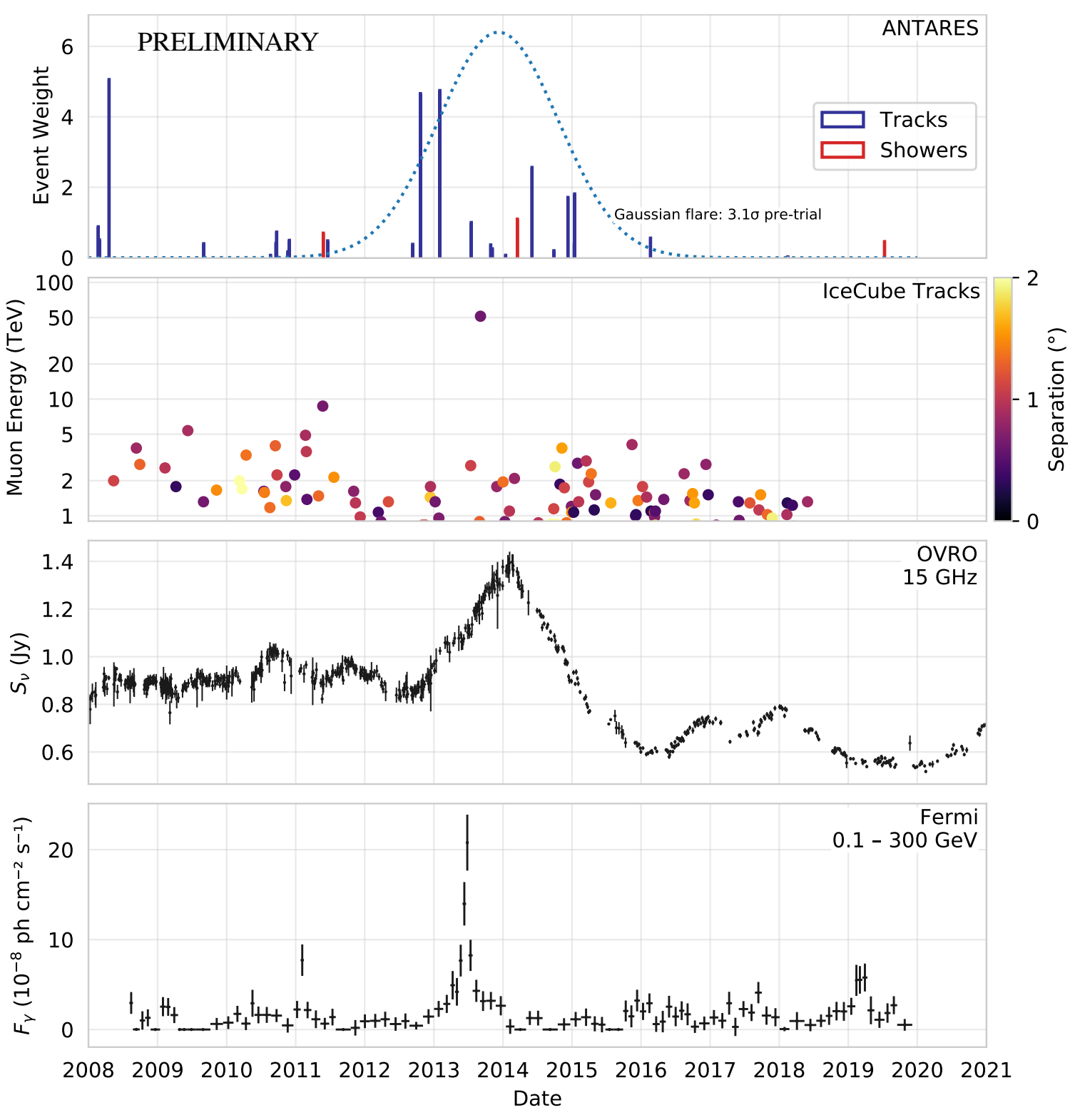

Figure 4: Multi-messenger light-curves from the direction of the blazar J0242+1101 as a function of time, since 2008. First plot: weighted time distribution of the ANTARES tracks (showers) within $5^{\circ}\left(10^{\circ}\right)$ from J0242+1101. The Gaussian profile has been drawn using the best-fit values of $\hat{\sigma}_{t}$ and $\hat{T}_{0}$ reported in Table 1 . Second plot: weighted time distribution of the IceCube tracks closer to J0242+1101 than their 50\% angular error. The applied weight corresponds to the energy of each event. The color scale indicates the event angular distance from the source. Third plot: OVRO radio light-curve for J0242+1101. Fourth plot: adaptive binned $\gamma$-ray light-curve obtained from Fermi LAT data for J0242+1101. 


\section{Conclusions}

The result of a search for time and space clustering of ANTARES events from the direction of 2774 radio-selected blazars has been presented. The blazar list employed in this analysis corresponds to the most up-to-date version of the catalog for which a promising directional correlation with IceCube events has been recently reported $[4,5]$. The analysis method is based on an unbinned maximum likelihood approach, with generic Gaussian and Box profiles assumed for the signal time emission. No significant neutrino flare has been found in the search. The lowest pre-trial p-value is obtained for the blazar J1500-2358 using both time profiles, with a pre-trial significance of $3.3 \sigma(3.4 \sigma)$ obtained for the Gaussian-shape (Box-shape) assumption, corresponding to a post-trial p-value of $56 \%(40 \%)$. A pre-trial significance of over $3 \sigma$ for at least one of the tested time profile has been obtained for other six sources: J1517-4424, J1606+2717, J1418-3509, J0242+1101, J0732-0150, and J0641-3554. Finally, the remarkable case of the blazar J0242+1101, showing an intriguing overlap in time of the flaring emission in radio, $\gamma$-ray and neutrino, has been reported. The chance probability of the multi-messenger association is under study.

\section{References}

[1] IceCube Collaboration, C. Kopper and E. Blaufuss, GCN Circular 21916 (2017).

[2] IceCube, Fermi-LAT, MAGIC, AGILE, ASAS-SN, HAWC, HESS, INTEGRAL, Kanata, Kiso, Kapteyn, Liverpool Telescope, Subaru, Swift NuSTAR, VERITAS, VLA/17B-403 Collaboration, M. G. Aartsen et al., Science 361 (2018) eaat1378.

[3] IceCube Collaboration, M. G. Aartsen et al., Science 361 (2018) 147-151.

[4] A. V. Plavin, Y. Y. Kovalev, Y. A. Kovalev, and S. Troitsky, Astrophys. J. 894 (2020) 101.

[5] A. V. Plavin, Y. Y. Kovalev, Y. A. Kovalev, and S. V. Troitsky, Astrophys. J. 908 (2021) 157.

[6] ANTARES Collaboration, J. Aublin and A. Plavin, PoS(ICRC2021)1240 (these proceedings).

[7] ANTARES Collaboration, A. Albert et al., Astrophys. J. 911 (2021) 48.

[8] ANTARES Collaboration, G. Illuminati, PoS(ICRC2021)1142 (these proceedings).

[9] ANTARES Collaboration, A. Albert et al., Phys. Rev. D96 (2017) 082001.

[10] ANTARES Collaboration, A. Albert et al., Astrophys. J. 879 (2019) 108.

[11] IceCube Collaboration, C. Kopper, PoS(ICRC2017)981 (2017).

[12] IceCube Collaboration, J. Stettner, PoS(ICRC2019)1017 (2019).

[13] J. Braun, M. Baker, J. Dumm, et al., Astropart.Phys. 33 (2010) 175 - 181.

[14] J. L. Richards et al., Astrophys. J. Suppl. 194 (2011) 29.

[15] I. G. Kramarenko, A. B. Pushkarev, Y. Y. Kovalev, M. L. Lister, T. Hovatta, and T. Savolainen, MNRAS submitted (2021) arXiv:2106.08416.

[16] All-sky point-source IceCube data: years 2008-2018, https://icecube.wisc.edu/data-releases/2021/01/all-sky-point-source-icecube-data-years-2008-2018/. 


\section{Full Authors List: ANTARES Collaboration}

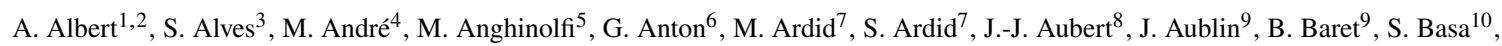
B. Belhorma ${ }^{11}$, M. Bendahman ${ }^{9}, 12$, V. Bertin ${ }^{8}$, S. Biagi ${ }^{13}$, M. Bissinger ${ }^{6}$, J. Boumaaza ${ }^{12}$, M. Bouta ${ }^{14}$, M.C. Bouwhuis ${ }^{15}$, H. Brânzaş ${ }^{16}$, R. Bruijn ${ }^{15,17}$, J. Brunner ${ }^{8}$, J. Busto ${ }^{8}$, B. Caiffi ${ }^{5}$, A. Capone ${ }^{18,19}$, L. Caramete ${ }^{16}$, J. Carr ${ }^{8}$, V. Carretero ${ }^{3}$, S. Celli ${ }^{18,19}$, M. Chabab $^{20}$, T. N. Chau ${ }^{9}$, R. Cherkaoui El Moursli ${ }^{12}$, T. Chiarusi ${ }^{21}$, M. Circella ${ }^{22}$, A. Coleiro ${ }^{9}$, M. Colomer-Molla ${ }^{9}, 3$, R. Coniglione ${ }^{13}$, P. Coyle ${ }^{8}$, A. Creusot $^{9}$, A. F. Díaz ${ }^{23}$, G. de Wasseige ${ }^{9}$, A. Deschamps ${ }^{24}$, C. Distefano ${ }^{13}$, I. Di Palma ${ }^{18,19}$, A. Domi ${ }^{15,17}$, C. Donzaud ${ }^{9,25}$, D. Dornic ${ }^{8}$, D. Drouhin ${ }^{1,2}$, T. Eberl ${ }^{6}$, T. van Eeden ${ }^{15}$, D. van Eijk ${ }^{15}$, N. El Khayati ${ }^{12}$, A. Enzenhöfer ${ }^{8}$, P. Fermani ${ }^{18,19}$, G. Ferrara $^{13}$, F. Filippini ${ }^{21,26}$, L.A. Fusco ${ }^{8}$, Y. Gatelet ${ }^{9}$, P. Gay ${ }^{27,9}$, H. Glotin $^{28}$, R. Gozzini ${ }^{3}$, R. Gracia Ruiz ${ }^{15}$, K. Graf ${ }^{6}$, C. Guidi ${ }^{5,29}$, S. Hallmann ${ }^{6}$, H. van Haren ${ }^{30}$, A.J. Heijboer ${ }^{15}$, Y. Hello ${ }^{24}$, J.J. Hernández-Rey ${ }^{3}$, J. Höß1 ${ }^{6}$, J. Hofestädt ${ }^{6}$, F. Huang ${ }^{8}$, G. Illuminati ${ }^{9}, 21,26$, C.W James ${ }^{31}$, B. Jisse-Jung ${ }^{15}$, M. de Jong ${ }^{15,32}$, P. de Jong ${ }^{15}$, M. Kadler ${ }^{33}$, O. Kalekin ${ }^{6}$, U. Katz ${ }^{6}$, N.R. Khan-Chowdhury ${ }^{3}$, A. Kouchner ${ }^{9}$, Y. Y. Kovalev $^{44,45,46}$, Yu. A. Kovalev ${ }^{44}$, I. Kreykenbohm ${ }^{34}$, V. Kulikovskiy ${ }^{5,36}$, R. Lahmann ${ }^{6}$, R. Le Breton ${ }^{9}$, D. Lefèvre ${ }^{35}$, E. Leonora ${ }^{36}$, G. Levi ${ }^{21,26}$, M. Lincetto ${ }^{8}$, D. Lopez-Coto ${ }^{37}$, S. Loucatos ${ }^{38,9}$, L. Maderer ${ }^{9}$, J. Manczak ${ }^{3}$, M. Marcelin 10, A. Margiotta ${ }^{21,26}$, A. Marinelli ${ }^{39}$, J.A. Martínez-Mora ${ }^{7}$, K. Melis ${ }^{15,17}$, P. Migliozzi ${ }^{39}$, A. Moussa ${ }^{14}$, R. Muller ${ }^{15}$, L. Nauta ${ }^{15}$, S. Navas ${ }^{37}$, E. Nezri ${ }^{10}$, B. O'Fearraigh ${ }^{15}$, G.E. Păvălaș ${ }^{16}$, C. Pellegrino ${ }^{21,40,41}$, M. Perrin-Terrin ${ }^{8}$, V. Pestel ${ }^{15}$, P. Piattelli ${ }^{13}$, C. Pieterse ${ }^{3}$, A. V. Plavin ${ }^{44,45}$, A. Păun ${ }^{16}$, C. Poirè, V. Popa ${ }^{16}$, T. Pradier ${ }^{1}$, A. B. Pushkarev ${ }^{48,44,45}$, N. Randazzo ${ }^{36}$, S. Reck ${ }^{6}$, G. Riccobene ${ }^{13}$, A. Romanov ${ }^{5,29}$, A. Sánchez-Losa ${ }^{3,22}$, F. Salesa Greus ${ }^{3}$, D. F. E. Samtleben ${ }^{15,32}$, M. Sanguineti ${ }^{5,29}$, P. Sapienza ${ }^{13}$, J. Schnabel ${ }^{6}$, J. Schumann ${ }^{6}$, F. Schüssler ${ }^{38}$, M. Spurio ${ }^{21,26}$, Th. Stolarczyk ${ }^{38}$, M. Taiuti ${ }^{5,29}$, Y. Tayalati ${ }^{12}$, S.J. Tingay ${ }^{31}$, S. V. Troitsky ${ }^{47}$, B. Vallage ${ }^{38,9}$, V. Van Elewyck ${ }^{9,41}$, F. Versari ${ }^{21,26,9}$, S. Viola ${ }^{13}$, D. Vivolo ${ }^{39,43}$, J. Wilms ${ }^{34}$, S. Zavatarelli ${ }^{5}$, A. Zegarelli ${ }^{18,19}$, J.D. Zornoza ${ }^{3}$, and J. Zúñiga ${ }^{3}$

${ }^{1}$ Université de Strasbourg, CNRS, IPHC UMR 7178, F-67000 Strasbourg, France. ${ }^{2}$ Université de Haute Alsace, F-68100 Mulhouse, France. ${ }^{3}$ IFIC - Instituto de Física Corpuscular (CSIC - Universitat de València) c/ Catedrático José Beltrán, 2 E-46980 Paterna, Valencia, Spain. ${ }^{4}$ Technical University of Catalonia, Laboratory of Applied Bioacoustics, Rambla Exposició, 08800 Vilanova i la Geltrú, Barcelona, Spain. ${ }^{5}$ INFN - Sezione di Genova, Via Dodecaneso 33, 16146 Genova, Italy. ${ }^{6}$ Friedrich-Alexander-Universität Erlangen-Nürnberg, Erlangen Centre for Astroparticle Physics, Erwin-Rommel-Str. 1, 91058 Erlangen, Germany. ${ }^{7}$ Institut d'Investigació per a la Gestió Integrada de les Zones Costaneres (IGIC) - Universitat Politècnica de València. C/ Paranimf 1, 46730 Gandia, Spain. ${ }^{8}$ Aix Marseille Univ, CNRS/IN2P3, CPPM, Marseille, France. ${ }^{9}$ Université de Paris, CNRS, Astroparticule et Cosmologie, F-75013 Paris, France. ${ }^{10}$ Aix Marseille Univ, CNRS, CNES, LAM, Marseille, France. ${ }^{11}$ National Center for Energy Sciences and Nuclear Techniques, B.P.1382, R. P.10001 Rabat, Morocco. ${ }^{12}$ University Mohammed V in Rabat, Faculty of Sciences, 4 av. Ibn Battouta, B.P. 1014, R.P. 10000 Rabat, Morocco. ${ }^{13}$ INFN - Laboratori Nazionali del Sud (LNS), Via S. Sofia 62, 95123 Catania, Italy. ${ }^{14}$ University Mohammed I, Laboratory of Physics of Matter and Radiations, B.P.717, Oujda 6000, Morocco. ${ }^{15}$ Nikhef, Science Park, Amsterdam, The Netherlands. ${ }^{16}$ Institute of Space Science, RO-077125 Bucharest, Măgurele, Romania. ${ }^{17}$ Universiteit van Amsterdam, Instituut voor Hoge-Energie Fysica, Science Park 105, 1098 XG Amsterdam, The Netherlands. ${ }^{18}$ INFN - Sezione di Roma, P.le Aldo Moro 2, 00185 Roma, Italy. ${ }^{19}$ Dipartimento di Fisica dell'Università La Sapienza, P.le Aldo Moro 2, 00185 Roma, Italy. ${ }^{20}$ LPHEA, Faculty of Science - Semlali, Cadi Ayyad University, P.O.B. 2390, Marrakech, Morocco. ${ }^{21}$ INFN - Sezione di Bologna, Viale Berti-Pichat 6/2, 40127 Bologna, Italy. ${ }^{22}$ INFN - Sezione di Bari, Via E. Orabona 4, 70126 Bari, Italy. ${ }^{23}$ Department of Computer Architecture and Technology/CITIC, University of Granada, 18071 Granada, Spain. ${ }^{24}$ Géoazur, UCA, CNRS, IRD, Observatoire de la Côte d'Azur, Sophia Antipolis, France. ${ }^{25}$ Université Paris-Sud, 91405 Orsay Cedex, France. ${ }^{26}$ Dipartimento di Fisica e Astronomia dell'Università, Viale Berti Pichat 6/2, 40127 Bologna, Italy. ${ }^{27}$ Laboratoire de Physique Corpusculaire, Clermont Université, Université Blaise Pascal, CNRS/IN2P3, BP 10448, F-63000 Clermont-Ferrand, France. ${ }^{28}$ LIS, UMR Université de Toulon, Aix Marseille Université, CNRS, 83041 Toulon, France. ${ }^{29}$ Dipartimento di Fisica dell'Università, Via Dodecaneso 33, 16146 Genova, Italy. ${ }^{30}$ Royal Netherlands Institute for Sea Research (NIOZ), Landsdiep 4, 1797 SZ 't Horntje (Texel), the Netherlands. ${ }^{31}$ International Centre for Radio Astronomy Research - Curtin University, Bentley, WA 6102, Australia. ${ }^{32}$ Huygens-Kamerlingh Onnes Laboratorium, Universiteit Leiden, The Netherlands. ${ }^{33}$ Institut für Theoretische Physik und Astrophysik, Universität Würzburg, Emil-Fischer Str. 31, 97074 Würzburg, Germany. ${ }^{34}$ Dr. Remeis-Sternwarte and ECAP, Friedrich-Alexander-Universität Erlangen-Nürnberg, Sternwartstr. 7, 96049 Bamberg, Germany. ${ }^{35}$ Mediterranean Institute of Oceanography (MIO), Aix-Marseille University, 13288, Marseille, Cedex 9, France; Université du Sud Toulon-Var, CNRS-INSU/IRD UM 110, 83957, La Garde Cedex, France. ${ }^{36}$ INFN - Sezione di Catania, Via S. Sofia 64, 95123 Catania, Italy. ${ }^{37}$ Dpto. de Física Teórica y del Cosmos \& C.A.F.P.E., University of Granada, 18071 Granada, Spain. ${ }^{38}$ IRFU, CEA, Université Paris-Saclay, F-91191 Gif-sur-Yvette, France. ${ }^{39}$ INFN - Sezione di Napoli, Via Cintia 80126 Napoli, Italy. ${ }^{40}$ Museo Storico della Fisica e Centro Studi e Ricerche Enrico Fermi, Piazza del Viminale 1, 00184, Roma. ${ }^{41}$ INFN - CNAF, Viale C. Berti Pichat 6/2, 40127, Bologna. ${ }^{42}$ Institut Universitaire de France, 75005 Paris, France. ${ }^{43}$ Dipartimento di Fisica dell'Università Federico II di Napoli, Via Cintia 80126, Napoli, Italy. ${ }^{44}$ Lebedev Physical Institute of the Russian Academy of Sciences, Leninsky prospekt 53, 119991 Moscow, Russia ${ }^{45}$ Moscow Institute of Physics and Technology, Institutsky per. 9, Dolgoprudny, Moscow region, 141700, Russia. ${ }^{46}$ Max-Planck-Institut für Radioastronomie, Auf dem Hügel 69, 53121 Bonn, Germany. ${ }^{47}$ Institute for Nuclear Research, 60th October anniversary prospect 7a, Moscow 117312, Russia. ${ }^{48}$ Crimean Astrophysical Observatory, Nauchny 298409, Crimea, Russia

\section{OVRO Collaboration}

A. C. S. Readhead ${ }^{49}$, T. Hovatta ${ }^{50,51}$, S. Kiehlmann ${ }^{52,53}$. 
49 Owens Valley Radio Observatory, California Institute of Technology, Pasadena, CA 91125, USA. ${ }^{50}$ Finnish Centre for Astronomy with ESO, University of Turku, FI-20014 Turku, Finland. ${ }^{51}$ Aalto University Metsähovi Radio Observatory, Metsähovintie 114, FI-02540 Kylmälä, Finland. ${ }^{52}$ Institute of Astrophysics, Foundation for Research and Technology-Hellas, GR-71110 Heraklion, Greece. ${ }^{53}$ Department of Physics, Univ. of Crete, GR-70013 Heraklion, Greece. 